\title{
Dislocation Arrangement in a Thick LEO GaN Film on Sapphire
}

\author{
Kathleen A. Dunn*, Susan E. Babcock*, Donald S. Stone*, Richard J. Matyi*, Ling \\ Zhang $\dagger$ and Thomas F. Kuech $\dagger$ \\ *Materials Science and Engineering ; †Chemical Engineering \\ University of Wisconsin - Madison, Madison, WI 53706
}

\begin{abstract}
Diffraction-contrast TEM, focused probe electron diffraction, and high-resolution $\mathrm{X}$-ray diffraction were used to characterize the dislocation arrangements in a $16 \mu \mathrm{m}$ thick coalesced GaN film grown by MOVPE LEO. As is commonly observed, the threading dislocations that are duplicated from the template above the window bend toward (0001). At the coalescence plane they bend back to lie along [0001] and thread to the surface. In addition, three other sets of dislocations were observed. The first set consists of a wall of parallel dislocations lying in the coalescence plane and nearly parallel to the substrate, with Burgers vector (b) in the (0001) plane. The second set is comprised of rectangular loops with $\mathbf{b}=1 / 3$ [11 20 ] (perpendicular to the coalescence boundary) which originate in the coalescence boundary and extend laterally into the film on the $(1 \overline{100})$. The third set of dislocations threads laterally through the film along the $\left[\begin{array}{l}1 \\ 100\end{array}\right]$ bar axis with $1 / 3<11 \overline{2} 0>$-type Burgers vectors These sets result in a dislocation density of $\sim 10^{9} \mathrm{~cm}^{-2}$. High resolution X-ray reciprocal space maps indicate wing tilt of $\sim 0.5^{\circ}$.
\end{abstract}

\section{INTRODUCTION}

High dislocation densities plague $\mathrm{GaN}$ films because the commercially available bulk substrates all have substantial lattice mismatch (13.5\% for $\mathrm{Al}_{2} \mathrm{O}_{3}, 3.3 \%$ for $\left.\mathrm{SiC}\right)$ and thermal expansion coefficient (TEC) mismatch with $\mathrm{GaN}$ (34\% and $25 \%$ for $\mathrm{Al}_{2} \mathrm{O}_{3}$ and $\mathrm{SiC}$, respectively). Presumably, eliminating one or both of these sources of mechanical stress is a step towards reducing the defect densities. Lateral epitaxial overgrowth (LEO) relieves components of the lattice mismatch stress by growing part of the GaN film over an amorphous material $[1,2]$. This report describes the dislocation microstructure in a $16 \mu \mathrm{m}$ thick coalesced film grown by the LEO method on a sapphire substrate. TEM images of the entire thickness of the film over five periods of the LEO mask pattern reveal a complex but characteristic arrangement of dislocations, many of which appear not to have their origin in the template.

\section{EXPERIMENTAL PROCEDURES}

The LEO substrate was fabricated using conventional CVD processes to grow a $1 \mu \mathrm{m}$ thick GaN template layer (MOCVD with TMGa and ammonia) and a $\sim 0.1 \mu \mathrm{m}$ thick $\mathrm{SiO}_{2}$ glass mask layer. Openings $2 \mu \mathrm{m}$ wide, oriented along $\left[\begin{array}{ll}1 & \overline{1} 00\end{array}\right]_{\mathrm{GaN}}$ and separated by $10 \mu \mathrm{m}$ of glass, were patterned into the mask using conventional lithography. A $16 \mu \mathrm{m}$ thick LEO film was grown at $1100^{\circ} \mathrm{C}$ in two hours by MOVPE using diethyl-GaCl and ammonia as precursors with a V/III ratio of 1800 and hydrogen carrier, as described in [3]. Cross-sectional TEM specimens were prepared by tripod polishing with final thinning by Ar ion milling. Two-beam and weak-beam diffraction contrast TEM were used to image the dislocation arrangements in the film and determine Burgers vectors of the various sets of dislocations observed. Convergent probe microdiffraction and high- 
resolution x-ray diffraction reciprocal space maps of the symmetric 0002 reflection [4] were used to investigate the extent of lattice tilt in the LEO material.

\section{RESULTS}

Prior to coalescence, the cross-section of the growing LEO material was rectangular with smooth, vertical sidewalls on (11 20) that terminated in small, inclined bevels at the top surface. The bevels remained after coalescence, leaving $\sim 2 \mu \mathrm{m}$ deep Vshaped trenches where the coalescence boundaries intersect the surface.

High-resolution $\mathrm{x}$-ray reciprocal space maps were recorded using the 0002 point with the stripes oriented both perpendicular (Fig 1a) and parallel (Fig 1b) to the diffractometer plane. The appearance of three distinct peaks in Fig 1a, but not 1b, indicates that the c-axis is tilted $\pm 0.5^{\circ}$ about the stripe axis in a portion of the material, presumably over the glass [5]. The downward shift of the side peaks corresponds to a relative expansion of the lattice along [0001] in the regions where the c-axis is tilted, a result that is consistent with different levels of in-plane compressive stress existing in the tilted and untilted regions of the material. The significant difference in the diffuse scatter between the perpendicular and parallel maps suggests a highly anisotropic defect structure.

Figure 2a is a montage of TEM images showing four periods of the LEO structure. The schematic in Fig. 2b illustrates the components of the characteristic dislocation arrangement that are repeated in each period. Labeled with Roman numerals in Figs. 2a $\&$ b are: (I) a core of threading dislocations (TDs) that are copied from the template, (II) threading and longitudinally oriented dislocations in the coalescence plane (CP), (III) a $\mathrm{V}$-shaped complex of dislocation loops, and (IV) longitudinal dislocations that run along the window axis in the upper part of the film. The arrows in the collection of unit cells in Fig $2 \mathrm{c}$ indicate the Burgers vectors (b) for each of these families of dislocations. The threading dislocations in the core have all of the "a" and "a+c" type Burgers vectors that typically populate epitaxial GaN films. The Burgers vectors of the dislocations in

(a)

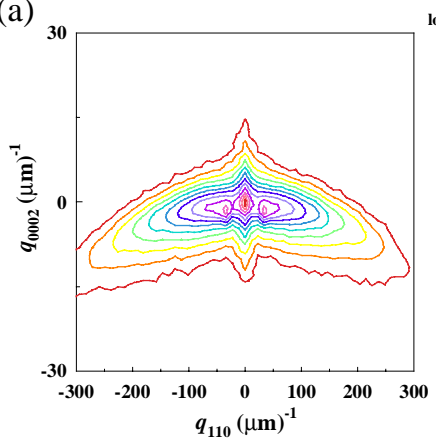

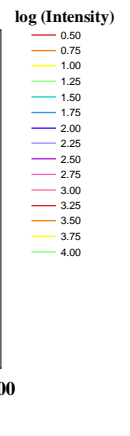

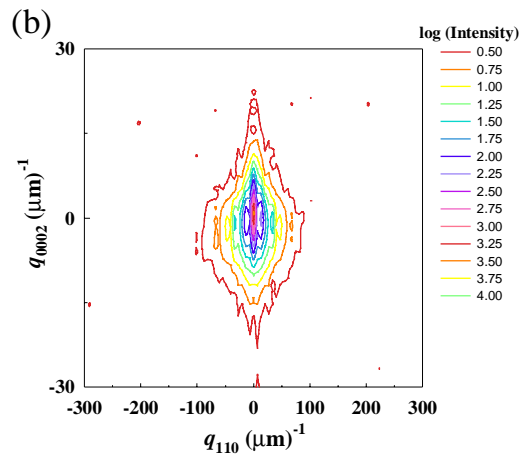

(b)

Figure 1. High resolution $X$-ray diffraction reciprocal space maps recorded with the stripes perpendicular (Fig 1a) and parallel (Fig 1b) to the diffractometer plane. The three peaks in (a) indicate three distinct orientations for the c-axis, while the difference in the distribution of diffuse scatter between $(a)$ and $(b)$ indicates a highly anisotropic dislocation structure. 


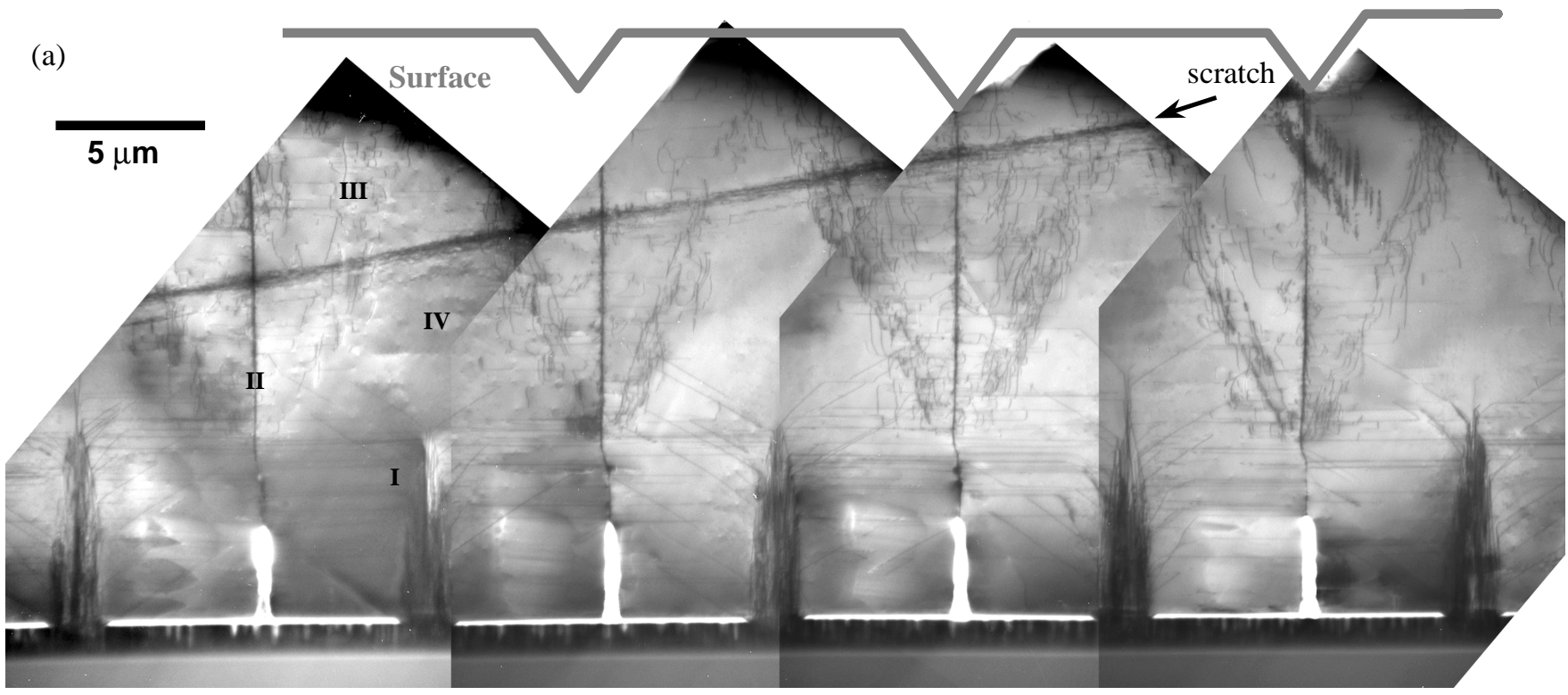

Figure 2. (a) Composite TEM image showing nearly four periods of the LEO structure. Four families of dislocations are labeled: (I) threading dislocations (TDs) copied from the template, (II) dislocations in the coalescence plane, (III) a Vshaped arrangement of rectangular loops, and (IV) longitudinal dislocations lying along the stripe axis. 


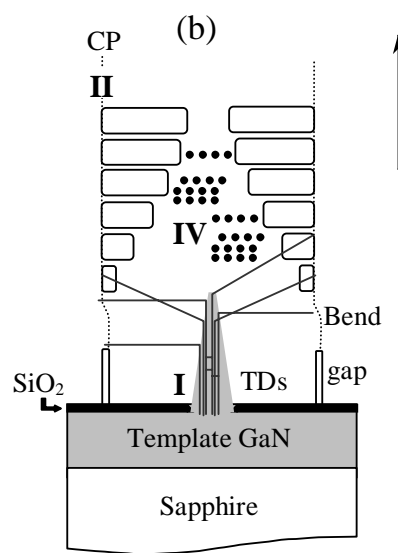

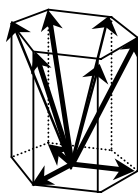

$\mathbf{I}$

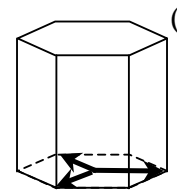

II

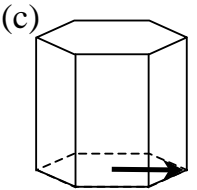

III

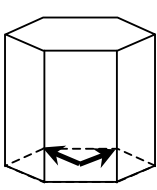

IV

Figure 2 (b) Schematic illustrating the four families of dislocations identified in the film: (I) the core of threading dislocations, (II) coalescence plane dislocations, (III) rectangular loops, and (IV) longitudinal dislocations. (c) Schematic illustrating the orientations of $\boldsymbol{b}$ for each set of dislocations.

each of the other families are a specific subset of these b's. Limitation to specific b's indicates that these are introduced into the microstructure to fulfill a particular purpose.

\section{(I) Threading Dislocations}

Threading dislocations are copied from the underlying template in the region above the window. In this sample, within $8 \mu \mathrm{m}$ of growth all the threading dislocations bend away from [0001]. All appear to bend in the plane normal to the stripe axis. Some, but not all, bend $90^{\circ}$ to lie on (0001); others prefer one of several other habit planes [6]. As they approach the coalescence plane, at least a subset, if not all, of these dislocations bend again to thread to the surface in the coalescence plane (Fig. 3a). Even if there is no crystallographic tilt, net $\mathbf{b}$, or disregistry at the coalescence plane, there is a dislocation structure in the boundary as a result of the bending threading dislocations. These dislocations do not disappear into the interface, nor do they continue into the next LEO stripe. They could act as sources or sinks for dislocations and point defects and/or provide a mechanism for mechanical deformation at the coalescence plane.

\section{(II) Coalescence plane}

In addition to the displaced threading dislocations, there is a wall of parallel dislocations (Fig 3b) that lie parallel or slightly inclined to the substrate and have Burgers vectors in the basal plane. These dislocations are present in somewhat dense sets with a local spacing that varies from 40-190 $\mathrm{nm}$ along the boundary. Their configurations are reminiscent of dislocation pile-ups in plastically deformed materials. The local dislocation spacings in this segment of the boundary correspond to a lower and upper estimate for the local misorientation across the coalescence plane of $0.16^{\circ}$ and $0.43^{\circ}$, assuming $\mathbf{b}=1 / 3\left[\begin{array}{ll}11 & \overline{2} 0\end{array}\right]$ for all of the dislocations. These angles will be $13 \%$ smaller if the b's are $1 / 3[\overline{2} 110]$ or $1 / 3[1 \overline{2} 10]$. Focused probe (micro-) diffraction, a technique with $\sim 0.5^{\circ}$ sensitivity, revealed no discernable tilt across this coalescence plane, consistent with the very small misorientation calculated from the dislocation spacing. These preliminary measurements were made near the top surface of the film. The inexact agreement with the $\mathrm{x}$-ray diffraction data concerning the magnitude of the tilt (the x-ray results suggest $\sim 1^{\circ}$ of tilt across the coalescence plane) reaffirms the inhomogeneous nature of the local tilt throughout the sample. 

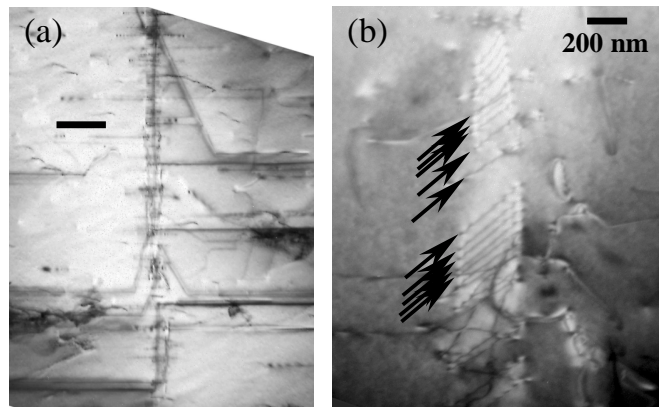

Figure 3. Structure of the coalescence plane. (a) Threading dislocations are displaced from the core to thread to the surface along the CP. (b) Walls of dislocations that lie in the $C P$ with line direction approximately parallel to the substrate have configurations reminiscent of dislocation pile-ups in plastically deformed materials.

\section{(III) Rectangular loops}

The third set of dislocations consists of rectangular loops in the (1 $\overline{100})$ plane, which give rise to the distinctive $\mathrm{V}$-shaped arrangement of edge-type dislocations that is centered on each coalescence plane (Figs. 2 and 4). The configuration of the loops, particularly near the apex where the entirety of the loop is visible (Fig. 4a), indicates that they form at the coalescence boundary and propagate into the LEO material. Closer to the surface of the film where the loops have propagated as far as $5 \mu \mathrm{m}$, only the vertical segments of the loops are clearly visible in many instances. Considering that the TEM specimen is thin $(0.1-0.2 \mu \mathrm{m}$ thick $)$, that the loop could lie anywhere through the specimen thickness, and that the sample may not be sectioned exactly on (1 $\overline{100})$, the observed images are consistent with rectangular loops in the $(1 \overline{100})$ plane. The dislocation images recorded under several different two-beam diffracting conditions were consistent with all of the loops having $b=1 / 3\left[\begin{array}{ll}11 & \overline{2} 0\end{array}\right]$, the a-type Burgers vector that lies perpendicular to the coalescence plane. The reversal of the characteristic black-whiteblack $\mathbf{g} \cdot \mathbf{b}=2$ contrast to white-black-white contrast at the coalescence plane indicates that the direction of the Burgers vector is opposite in the two legs of the "V." Due to the presence of the glass, the lattice parameter in this direction is not constrained by the substrate. Thus, these dislocations do not accommodate lattice mismatch, but rather must form in response to some other mechanical stress.

\section{(IV) Longitudinal dislocations}

The regions above the core of threading dislocations and between the legs of neighboring "V's" are populated by longitudinal dislocations of the type that have been observed in a number of films [7]. These dislocations have a-type Burgers vectors. The contrast produced where they intersect the surface of the TEM sample suggests that they have a screw component (i.e., Burgers vector other than that of the loops), but additional study of plan view samples is needed to characterize them more completely.

\section{DISCUSSION}

Four distinct dislocation families were observed in a thick LEO GaN film grown on sapphire. Each family has specific Burgers vectors, suggesting that they are introduced into the microstructure for a specific reason. The orientation of the Burgers vector in one of the dominant families, the rectangular dislocation loops (III), suggests that they form to in response to stresses other than lattice mismatch. An obvious candidate is TEC mismatch. An analysis of the deformation of the crystal that would be produced by the 


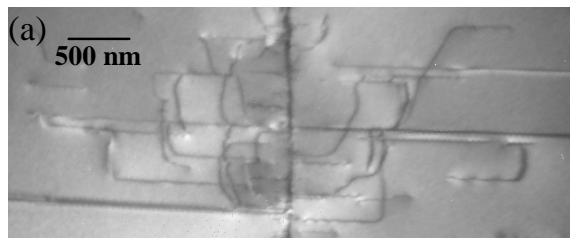

Figure 4. TEM images showing lateral extent of rectangular dislocation loops. Notice the contrast reversal of the dislocation image when the coalescence plane is crossed.

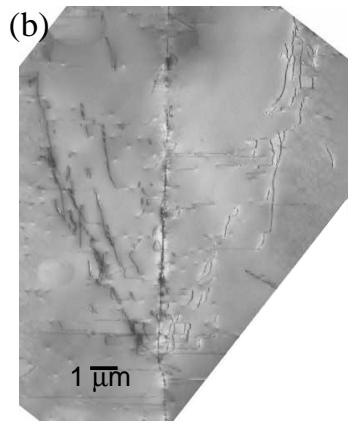

dislocation loops alone shows their net effect is to cause a contraction of the material in the bar by buckling it like an accordion. Other dislocations would be needed to satisfy compatibility requirements caused by the $\mathrm{V}$-shaped configuration of the loops. Arguments can be made that the longitudinal dislocations in the coalescence plane and the LEO material satisfy these requirements, but further Burgers vector analysis and examination of the driving force would be needed to establish this mechanism unambiguously.

\section{CONCLUSIONS}

The dislocations observed in a thick, coalesced LEO GaN film on sapphire form a complex but characteristic arrangement, and do not appear to be solely copied from the template. While wing tilt and TD bending are commonly observed in LEO films, the observation of additional sets of dislocations with specific Burgers vectors suggests a large scale cooperative motion in response to the in-plane compressive stress or other driving forces.

\section{ACKNOWLEDGEMENTS}

This work was funded by the ONR-MURI on Compliant Substrates. Partial support for the electron microscopy facilities was provided by the NSF-MRSEC at UW-Madison.

\section{REFERENCES}

[1] T. S. Zheleva, O.-H. Nam, M. D. Bremser, and R. F. Davis, Appl. Phys. Lett. 71, 2472 (1997).

[2] D. Kapolnek, S. Keller, R. Vetury, R.D. Underwood, P. Kozodoy, S. P. DenBaars, and U.K. Mishra, Appl. Phys. Lett. 71, 1204 (1997).

[3] R. Zhang, L. Zhang, D. M. Hansen, M. P. Boleslawski, K. L. Chen, D. Q. Lu, B. Shen, Y.D. Zheng and T. F. Kuech, MRS Internet J. Nitride Semicond. Res. 4: U473-U478, Suppl. 1 (1999).

[4] B.K. Tanner and D. K. Bowen, High Resolution X-ray Diffractometry \& Topography, Taylor \& Francis, Bristol, PA 1998.

[5] P. Fini, L. Zhao, B. Moran, M. Hansen, H. Marchand, J. P. Ibbetson, S. P. DenBaars, U. K. Mishra, and J. S. Speck, Appl. Phys. Lett. 75(12), 1706 (1999).

[6] H. Marchand, X.H. Wu, J. P. Ibbetson, P.T. Fini, P. Kozodoy, S. Keller, J.S. Speck, S. P. DenBaars, U.K. Mishra, Appl. Phys. Lett. 73(6), 747 (1998).

[7] Z. Liliental-Weber, M. Benamara, W. Swider, J. Washburn, J. Park, P.A. Grudowski, C.J. Eiting, R.D. DupuisMRS Internet J. Nitride Semicond. Res. 4S1, G4.6 (1999). 